\title{
LIE SOLVABLE GROUP ALGEBRAS OF DERIVED LENGTH THREE
}

\author{
Menna Sahai
}

\begin{abstract}
Let $K$ be a field of characteristic $p>2$ and let $G$ be a group. Necessary and sufficient conditions are obtained so that the group algebra $K G$ is strongly Lie solvable of derived length at most 3 . It is also shown that these conditions are equivalent to $K G$ Lie solvable of derived length 3 in characteristic $p \geq 7$.
\end{abstract}

\section{Introduction}

Any associative ring $R$ gives rise to the associated Lie ring $L(R)$ under the Lie multiplication $[x, y]=x y-y x, x, y \in R$. We define, inductively, $\left[x_{1}, x_{2}, \ldots, x_{n}\right]=\left[\left[x_{1}, x_{2}, \ldots, x_{n-1}\right], x_{n}\right]$. An additive subgroup $V$ of $R$ is called a Lie ideal of $R$ if $[v, r] \in V$ for all $v \in V$ and $r \in R$. For any two Lie ideals $V$ and $W$, we denote by $[V, W]$ to be the additive subgroup of $R$ generated by $\{[v, w] \mid v \in V$ and $w \in W\}$.

We define the Lie derived series $\delta^{[n]}(L(R))$ and the strong Lie derived series $\delta^{(n)}(R), n \geq 0$, by induction as follows:

$$
\begin{aligned}
\delta^{[0]}(L(R)) & =\delta^{(0)}(R)=R, \\
\delta^{[n]}(L(R)) & =\left[\delta^{[n-1]}(L(R)), \delta^{[n-1]}(L(R))\right], \\
\delta^{[n]}(R) & =\left[\delta^{(n-1)}(R), \delta^{(n-1)}(R)\right] R .
\end{aligned}
$$

$R$ is Lie solvable of derived length $n$ if $\delta^{[n]}(L(R))=0$ but $\delta^{[n-1]}(L(R)) \neq$ 0 . Similarly $R$ is strongly Lie solvable of derived length $n$ if $\delta^{(n)}(R)=0$ but $\delta^{(n-1)}(R) \neq 0$. Lie solvable/strongly Lie solvable rings of derived length 2 are called Lie metabelian/strongly Lie metabelian rings. Also $R$ is said to be Lie centrally metabelian if $\left[\delta^{[2]}(L(R)), R\right]=0$.

Let $K$ be a field with Char $K=p$ and let $G$ be a group. It is known that the group algebra $K G$ is Lie solvable if and only if $G$ has a 2-abelian subgroup of index at most 2 , when $p=2$ and $G$ is $p$-abelian when $p \neq 2$. 
For $p \neq 2$ this is equivalent to $K G$ strongly Lie solvable (see [4, Chapter $\mathrm{V}]$ ). But the connection between the order of the derived subgroup $G^{\prime}$ of $G$ and the derived length of $K G$ is not known as yet. In this direction Levin and Rosenberger [1] have characterized Lie metabelian group rings. Lie centrally metabelian group algebras have been studied by Sharma and Srivastava [6] and Sahai and Srivastava $[3]$. It is shown in [1] that the group $\operatorname{ring} R G$ of a group $G$ over a commutative $\operatorname{ring} R$ is Lie metabelian if and only if it is strongly Lie metabelian.

In this paper, necessary and sufficient conditions for the group algebra $K G$, Char $K=p \geq 3$, to be strongly Lie solvable of derived length at most 3 have been obtained. It is shown that these conditions are equivalent to $K G$ Lie solvable of derived length at most 3 for $p \geq 7$.

\section{Results and Proofs}

Throughout this section $K$ denotes a field with Char $K=p \geq 3$ and $G$ denotes a group.

It is well known and easy to see that if $M$ and $N$ are normal subgroups of $G$, then

$$
\begin{aligned}
{[\Delta(M) K G, \Delta(N) K G] K G=} & \Delta((M, N)) K G \\
& +\Delta(M) \Delta(N) \Delta\left(G^{\prime}\right) K G \\
& +\Delta((M, G)) \Delta(N) K G \\
& +\Delta(M) \Delta((N, G)) K G .
\end{aligned}
$$

In particular, if we take $M=N=G^{\prime}$, then

$$
\begin{aligned}
\delta^{(2)}(K G)= & {\left[\Delta\left(G^{\prime}\right) K G, \Delta\left(G^{\prime}\right) K G\right] K G } \\
= & \Delta\left(G^{\prime \prime}\right) K G \\
& +\Delta\left(G^{\prime}\right)^{3} K G \\
& +\Delta\left(\gamma_{3}(G)\right) \Delta\left(G^{\prime}\right) K G \\
& +\Delta\left(G^{\prime}\right) \Delta\left(\gamma_{3}(G)\right) K G .
\end{aligned}
$$

Remark 2.1. If $G^{\prime}$ is central, then the above equation gives $\delta^{(2)}(K G)=\Delta\left(G^{\prime}\right)^{3} K G$. Further, if $\gamma_{3}(G)=G^{\prime}$, then $\delta^{(2)}(K G)=$ $\Delta\left(G^{\prime}\right)^{2} K G$.

If $|G|=p^{n}$, Char $K=p$ and $t(G)$ denotes the nilpotency index of the augmentation ideal $\Delta(G)$, then it is known that $n(p-1)+1 \leq t(G) \leq p^{n}$ with equality on the left/right hand side if and only if $G$ is elementary abelian/cyclic (see [2]). This will be used for $t\left(G^{\prime}\right)$, as $G^{\prime}$ is a finite $p$-group if $K G$ is Lie solvable. For any element $x \in G^{\prime}$ we denote $\hat{x}=$ $1+x+x^{2}+\cdots+x^{n-1}$ where order of $x$ is $n$.

We start with the following straightforward observation. 
Lemma 2.2. For all $n \geq 1, \Delta\left(G^{\prime}\right)^{2^{n}-1} K G \subseteq \delta^{(n)}(K G) \subseteq$ $\Delta\left(G^{\prime}\right)^{2^{n-1}} K G$.

Proof: The right hand side inclusion is immediate by induction on $n$. Since $\delta^{(1)}(K G)=\Delta\left(G^{\prime}\right) K G$ and the identity

$$
\delta_{1} \delta_{2}\left[g_{1}, g_{2}\right]=\left[\delta_{1} g_{1}, \delta_{2} g_{2}\right]-\left[\delta_{1}, \delta_{2} g_{2}\right] g_{1}-\left[\delta_{1} g_{1}, \delta_{2}\right] g_{2}+\left[\delta_{1}, \delta_{2}\right] g_{1} g_{2}
$$

is true for all $\delta_{1}, \delta_{2} \in \Delta\left(G^{\prime}\right)^{2^{n}-1}$ and $g_{1}, g_{2} \in G$, we have

$$
\begin{aligned}
\Delta\left(G^{\prime}\right)^{2^{n+1}-1} K G & =\Delta\left(G^{\prime}\right)^{2^{n}-1} \Delta\left(G^{\prime}\right)^{2^{n}-1} \Delta\left(G^{\prime}\right) K G \\
& =\Delta\left(G^{\prime}\right)^{2^{n}-1} \Delta\left(G^{\prime}\right)^{2^{n}-1}[K G, K G] K G \\
& \subseteq\left[\Delta\left(G^{\prime}\right)^{2^{n}-1} K G, \Delta\left(G^{\prime}\right)^{2^{n}-1} K G\right] K G \\
& \subseteq\left[\delta^{(n)}(K G), \delta^{(n)}(K G)\right] K G \\
& =\delta^{(n+1)}(K G) .
\end{aligned}
$$

This proves the left hand side inclusion by induction on $n$.

Theorem 2.3. Let $K$ be a field of characteristic $p \neq 2$ and let $G$ be a group. Then $\delta^{(3)}(K G)=0$ if and only if one of the following holds:

(i) $G$ is abelian.

(ii) $p=7, G^{\prime}=C_{7}$ and $\gamma_{3}(G)=1$.

(iii) $p=5, G^{\prime}=C_{5}$ and either $\gamma_{3}(G)=1$ or $\gamma_{n}(G)=G^{\prime}$ for all $n \geq 3$ with $x^{g}=x^{-1}$ for all $x \in G^{\prime}$ and for all $g \notin C_{G}\left(G^{\prime}\right)$.

(iv) $p=3, G^{\prime}$ is a group of one of the following types:

(a) $G^{\prime}=C_{3}$.

(b) $G^{\prime}=C_{3} \times C_{3}$ and either $\gamma_{3}(G)=1$ or $\gamma_{3}(G)=C_{3}, \gamma_{4}(G)=1$ or $\gamma_{n}(G)=G^{\prime}$, for all $n \geq 3$ with $x^{g}=x^{-1}$ for all $x \in G^{\prime}$ and for all $g \notin C_{G}\left(G^{\prime}\right)$.

(c) $G^{\prime}=C_{3} \times C_{3} \times C_{3}, \gamma_{3}(G)=1$.

Proof: Suppose that $\delta^{(3)}(K G)=0$. Since Char $K \neq 2, G^{\prime}$ is a finite p-group. Let $\left|G^{\prime}\right|=p^{n}$. By Lemma 2.2, $\Delta\left(G^{\prime}\right)^{7} K G \subseteq \delta^{(3)}(K G) \subseteq$ $\Delta\left(G^{\prime}\right)^{4} K G$. Thus $\Delta\left(G^{\prime}\right)^{7}=0$. This in turn implies $t\left(G^{\prime}\right) \leq 7$. By the discussion following just after Remark 2.1, we conclude that

(i) $p \geq 11$ implies $n=0$. In this case $G^{\prime}=1$ and thus $G$ is abelian.

(ii) $p=7$ and $G$ is non-abelian implies $n=1$ and $G^{\prime}=C_{7}$.

(iii) $p=5$ and $G$ is non-abelian implies $n=1$ and $G^{\prime}=C_{5}$.

(iv) $p=3$ and $G$ is non-abelian implies $G^{\prime}$ is $C_{3}$ or $C_{3} \times C_{3}$ or $C_{3} \times$ $C_{3} \times C_{3}$. 
If $G$ is abelian, we are through. So we discuss each non-abelian case separately.

Case (ii). $p=7$. In this case $G^{\prime}=C_{7}$. We shall show that $G^{\prime}$ is central, i.e., $\gamma_{3}(G)=1$. If not then $\gamma_{3}(G)=G^{\prime}$ and by Remark 2.1, $\delta^{(2)}(K G)=\Delta\left(G^{\prime}\right)^{2} K G$. Let $G^{\prime}=\langle x\rangle$. Then $(x-1)^{6}=\hat{x}$. Now for any $g \in G$, we have

$$
\begin{aligned}
0 & =\left[(x-1)^{2},(x-1)^{2} g^{-1}\right] \\
& =(x-1)^{3}\left[x, g^{-1}\right]+(x-1)^{2}\left[x, g^{-1}\right](x-1) \\
& =-x\left\{(x-1)^{3}((x, g)-1)+(x-1)^{2}((x, g)-1)\left(x^{g}-1\right)\right\} g^{-1} .
\end{aligned}
$$

If $(x, g)=x^{k}, 1 \leq k \leq 5$, then we get

$$
(x-1)^{4}\left(1+x+x^{2}+\cdots+x^{k-1}\right)\left(2+x+x^{2}+\cdots+x^{k}\right)=0 .
$$

Multiplying by $(x-1)^{2}$, we have $k(k+2) \hat{x}=0$. Thus $k(k+2)=0$ in $K$. So $k=5$. Now $k=5$ is not possible because otherwise

$$
(x-1)^{4}\left(1+x+x^{2}+x^{3}+x^{4}\right)\left(2+x+x^{2}+x^{3}+x^{4}+x^{5}\right)=0,
$$

which gives

$$
(x-1)^{5}\left(1+x+x^{2}+x^{3}+x^{4}\right)\left(5+4 x+3 x^{2}+2 x^{3}+x^{4}\right)=0 .
$$

Multiplying by $(x-1)$ we get $75 \hat{x}=0$ which is not true. Thus $k=0$ and $(x, g)=1$ for all $g \in G$. Hence $G^{\prime}$ is central.

Case (iii). $p=5$. In this case $G^{\prime}=C_{5}$. Let $G^{\prime}=\langle x\rangle$. If $G^{\prime}$ is not central, then $\gamma_{3}(G)=G^{\prime}$, and $\delta^{(2)}(K G)=\Delta\left(G^{\prime}\right)^{2} K G$. Let $(x, g)=x^{k}$ for some $g \in G, 1 \leq k \leq 3$. If we proceed exactly as in the previous case, we get $k(k+2)=0$ in $K$. This gives $k=3$. Thus $(x, g)=1$ or $x^{3}$, i.e., if $g \notin C_{G}\left(G^{\prime}\right)$, then $x^{g}=x^{-1}$, as desired.

Case (iv). $p=3$. If $G^{\prime}=C_{3}$, we are through. If $G^{\prime}=C_{3} \times C_{3}$ then $t\left(G^{\prime}\right)=5$ and $\gamma_{3}(G)=1$ or $C_{3}$ or $G^{\prime}$. If $\gamma_{3}(G)=1$, we are through. Consider the case when $\gamma_{3}(G)=C_{3}=\langle z\rangle$. Let $y \in G^{\prime}$ such that $y \notin \gamma_{3}(G)$. Since $\Delta\left(\gamma_{3}(G)\right) \Delta\left(G^{\prime}\right) K G \subseteq \delta^{(2)}(K G)$, we have that for all $g \in G$

$$
\begin{aligned}
0 & =\left[(z-1)(y-1) g^{-1},(z-1)(y-1)\right] \\
& =(z-1)^{2}(y-1)\left[g^{-1}, y\right]+(z-1)(y-1)\left[g^{-1}, z\right](y-1) \\
& =(z-1)^{2}(y-1) y((y, g)-1) g^{-1}+(z-1)(y-1) z((z, g)-1)\left(y^{g}-1\right) g^{-1} .
\end{aligned}
$$


First term is zero because $(y, g) \in \gamma_{3}(G)$ and $\Delta\left(\gamma_{3}(G)\right)^{3}=0$. Thus $(z-1)(y-1)\left(z^{k}-1\right)\left(y^{g}-1\right)=0$, where $(z, g)=z^{k}$. This implies that if $k \neq 0$, then $(z-1)^{2}(y-1)^{2}=0$, because $y^{g}=y(y, g)$ and $(y, g) \in \gamma_{3}(G)$. But this is a contradiction to the fact that $y \notin \gamma_{3}(G)$. Hence $k=0$. This shows that $\gamma_{3}(G)$ is central, i.e., $\gamma_{4}(G)=1$.

If $\gamma_{3}(G)=G^{\prime}$, then $\delta^{(2)}(K G)=\Delta\left(G^{\prime}\right)^{2} K G$. Let $G^{\prime}=\langle x\rangle \times\langle y\rangle$ and let $g \in G$ such that $g \notin C_{G}\left(G^{\prime}\right)$. Now $\left[(x-1)^{2},(x-1)^{2} g^{-1}\right]=0$. Using the fact that $(x-1)^{3}=0$ and expanding we get $(x-1)^{2}\left(x^{g}-1\right)^{2}=0$. This implies $x^{g} \in\langle x\rangle$. Similarly $y^{g} \in\langle y\rangle$. Suppose that $x^{g} \neq x$, so $x^{g}=x^{-1}$. If $y^{g}=y$, then $\left[(x-1)(y-1) g^{-1},(x-1)(y-1)\right]=0$ gives that $(x-1)^{2}(y-1)^{2}=0$. So $y \in\langle x\rangle$ which is not possible. Hence $y^{g} \neq y$ and we must have $y^{g}=y^{-1}$. Thus if $g \notin C_{G}\left(G^{\prime}\right)$, then $x^{g}=x^{-1}$ and $y^{g}=y^{-1}$ which proves that $u^{g}=u^{-1}$ for every $u \in G^{\prime}$.

Now if $G^{\prime}=C_{3} \times C_{3} \times C_{3}$, then $t\left(G^{\prime}\right)=7$. Also $\Delta\left(G^{\prime}\right)^{3}(K G) \subseteq$ $\delta^{(2)}(K G)$ and $\Delta\left(\gamma_{3}(G)\right) \Delta\left(G^{\prime}\right) K G \subseteq \delta^{(2)}(K G)$. We wish to prove that $G^{\prime}$ is central, i.e., $\gamma_{3}(G)=1$. Suppose, if possible $\gamma_{3}(G) \neq 1$. Let $1 \neq x \in \gamma_{3}(G)$. Choose $y, z \in G^{\prime}$ such that $G^{\prime}=\langle x\rangle \times\langle y\rangle \times\langle z\rangle$. As before for any $g \in G,\left[(x-1)^{2} g^{-1},(x-1)^{2}\right]=0$, implies $(x-1)^{2}\left(x^{g}-1\right)^{2}=$ 0 . This implies $x^{g} \in\langle x\rangle$, i.e., $x^{g}=x$ or $x^{-1}$. Next observe that for any $u, v \in G$, we have $\left[u^{-1}, v\right]=\left\{v^{u}-1-(v-1)\right\} u^{-1}$. We have $\left[(y-1)(z-1)^{2} g^{-1},(x-1)(z-1)\right]=0$ and so $(y-1)(z-1)^{2}\left(\left\{\left(x^{g}-1\right)-\right.\right.$ $\left.(x-1)\}\left(z^{g}-1\right)+(x-1)\left\{\left(z^{g}-1\right)-(z-1)\right\}\right)=0$. Let $z^{g}=x^{r} y^{s} z^{t}$. If $x^{g}=x$, we get $(y-1)(x-1)\left(x^{r} y^{s}-1\right) \hat{z}=0$. This is possible only if $x^{r} y^{s}=1$, because $x, y, z$ are independent. If $x^{g}=x^{-1}$, we get, after simplification that $(y-1)\left(x^{-1}-1\right)\left(x^{r} y^{s}-1\right) \hat{z}=0$ and hence again $x^{r} y^{s}=1$. Thus $z^{g} \in\langle z\rangle$. That is, $z^{g}=z$ or $z^{-1}$ for all $g \in G$. Further for any $g \in G,\left[(y-1)^{2}(z-1) g^{-1},(x-1)(z-1)\right]=0$ implies

$(y-1)^{2}(z-1)\left(\left\{\left(x^{g}-1\right)-(x-1)\right\}\left(z^{g}-1\right)+(x-1)\left\{\left(z^{g}-1\right)-(z-1)\right\}\right)=0$. If $z^{g}=z$ and $x^{g}=x^{-1}$, then we get $(y-1)^{2}(z-1)^{2}(x-1)=0$, which is impossible since $x, y, z$ are independent. Thus $z^{g}=z$ implies $x^{g}=x$. If $z^{g}=z^{-1}$ and $x^{g}=x$, we again get $(y-1)^{2}(z-1)^{2}(x-1)=0$, which is not possible as before. Finally if $z^{g}=z^{-1}$ and $x^{g}=x^{-1}$, we get $(y-1)^{2}(z-1)^{2}(x-1)\{x(z+1)+z\}=0$, which is again not possible because $x, y, z$ are independent. Thus for any $g \in G$, we have $z^{g}=z$ and $x^{g}=x$. Similarly for any $g \in G$, we must have $y^{g}=y$. Thus $G^{\prime}$ is central.

Now we prove the converse. If $G$ is abelian, then clearly $\delta^{(3)}(K G)=0$.

If Char $K=7$ and $G^{\prime}=C_{7}$ with $\gamma_{3}(G)=1$, then by Remark 2.1 $\delta^{(2)}(K G)=\Delta\left(G^{\prime}\right)^{3} K G$. Thus

$$
\begin{aligned}
\delta^{(3)}(K G) & =\left[\Delta\left(G^{\prime}\right)^{3} K G, \Delta\left(G^{\prime}\right)^{3} K G\right] K G \\
& \subseteq \Delta\left(G^{\prime}\right)^{6}[K G, K G] K G \subseteq \Delta\left(G^{\prime}\right)^{7} K G=0 .
\end{aligned}
$$


If Char $K=5$ and $G^{\prime}=C_{5}=\langle x\rangle$, say, then $t\left(G^{\prime}\right)=5$. First let $\gamma_{3}(G)=1$. Then as above $\delta^{(3)}(K G) \subseteq \Delta\left(G^{\prime}\right)^{7} K G=0$. Now let $\gamma_{3}(G)=$ $G^{\prime}$ with the condition that if $g \notin C_{G}\left(G^{\prime}\right)$, then $x^{g}=x^{-1}$. Clearly $\Delta\left(G^{\prime}\right) K G=(x-1) K G$ and by Remark $2.1, \delta^{(2)}(K G)=\Delta\left(G^{\prime}\right)^{2} K G$. Thus $\delta^{(3)}(K G)=\left[(x-1)^{2} K G,(x-1)^{2} K G\right] K G$. Let $g_{1}, g_{2} \in G$, then

$$
\begin{aligned}
{[} & \left.(x-1)^{2} g_{1},(x-1)^{2} g_{2}\right] \\
= & (x-1)^{4}\left[g_{1}, g_{2}\right]+(x-1)^{3}\left[g_{1}, x\right] g_{2}+(x-1)^{2}\left[g_{1}, x\right](x-1) g_{2} \\
& +(x-1)^{3}\left[x, g_{2}\right] g_{1}+(x-1)^{2}\left[x, g_{2}\right](x-1) g_{1} \\
= & (x-1)^{4}\left(\left(g_{1}^{-1}, g_{2}^{-1}\right)-1\right) g_{2} g_{1}+(x-1)^{3}\left(\left(g_{1}^{-1}, x^{-1}\right)-1\right) x g_{1} g_{2} \\
& +(x-1)^{2}\left(\left(g_{1}^{-1}, x^{-1}\right)-1\right) x\left(x^{g_{1}^{-1}}-1\right) g_{1} g_{2} \\
& +(x-1)^{3}\left(\left(x^{-1}, g_{2}^{-1}\right)-1\right) x^{g_{2}^{-1}} g_{2} g_{1} \\
& +(x-1)^{2}\left(\left(x^{-1}, g_{2}^{-1}\right)-1\right) x^{g_{2}^{-1}}\left(x^{g_{2}^{-1}}-1\right) g_{2} g_{1} \\
= & (x-1)^{2}\left(\left(g_{1}^{-1}, x^{-1}\right)-1\right) x\left((x-1)+\left(x^{g_{1}^{-1}}-1\right)\right) g_{1} g_{2} \\
& +(x-1)^{2}\left(\left(x^{-1}, g_{2}^{-1}\right)-1\right) x^{g_{2}^{-1}}\left((x-1)+\left(x^{g^{-1}}-1\right)\right) g_{2} g_{1} \\
= & (x-1)^{4}\left(\left(g_{1}^{-1}, x^{-1}\right)-1\right) g_{1} g_{2}+(x-1)^{4}\left(\left(x^{-1}, g_{2}^{-1}\right)-1\right) g_{2} g_{1}
\end{aligned}
$$

assuming that $x^{g_{1}}=x^{-1}, x^{g_{2}}=x^{-1}$, other cases give 0 . The expression on the right hand side is 0 as $t\left(G^{\prime}\right)=5$. Thus $\delta^{(3)}(K G)=0$.

If Char $K=3$ and $G^{\prime}=C_{3}$ then $t\left(G^{\prime}\right)=3$ and so $\delta^{(3)}(K G) \subseteq$ $\Delta\left(G^{\prime}\right)^{4} K G=0$.

Assume that $G^{\prime}=C_{3} \times C_{3}$. Then $t\left(G^{\prime}\right)=5$. Now if $\gamma_{3}(G)=1$ then $\delta^{(2)}(K G)=\Delta\left(G^{\prime}\right)^{3} K G$ and $\delta^{(3)}(K G) \subseteq \Delta\left(G^{\prime}\right)^{7} K G=0$. If $\gamma_{3}(G)=C_{3}$ and $\gamma_{4}(G)=1$, then

$$
\delta^{(2)}(K G)=\Delta\left(G^{\prime}\right)^{3} K G+\Delta\left(\gamma_{3}(G)\right) \Delta\left(G^{\prime}\right) K G .
$$

$\delta^{(3)}(K G)=0$ because $t\left(G^{\prime}\right)=5$ and $t\left(\gamma_{3}(G)\right)=3$. If $\gamma_{3}(G)=G^{\prime}$ and $u^{g}=u^{-1}$ for every $u \in G^{\prime}, g \notin C_{G}\left(G^{\prime}\right)$, assume that $G^{\prime}=\langle x\rangle \times\langle y\rangle$. Then $\Delta\left(G^{\prime}\right) K G=(x-1) K G+(y-1) K G$ and $\Delta\left(G^{\prime}\right)^{2} K G=(x-$ $1)^{2} K G+(y-1)^{2} K G+(x-1)(y-1) K G$. Also $\delta^{(2)}(K G)=\Delta\left(G^{\prime}\right)^{2} K G$. For any $g \notin C_{G}\left(G^{\prime}\right)$ and $u, v \in G^{\prime}$, we have

$$
\begin{aligned}
{[g,(u-1)(v-1)] } & =(u-1)[g, v]+[g, u](v-1) \\
& =(u-1)\left(\left(g^{-1}, v^{-1}\right)-1\right) v g+\left(\left(g^{-1}, u^{-1}\right)-1\right) u g(v-1) \\
& =(u-1)(v-1) v g+(u-1) u\left(v^{g^{-1}}-1\right) g \\
& =(u-1)(v-1)(v+u v+u) g \in \Delta\left(G^{\prime}\right)^{3} K G .
\end{aligned}
$$


Using this and above we see that $\delta^{(3)}(K G) \subseteq \Delta\left(G^{\prime}\right)^{5} K G=0$.

The case when $G^{\prime}=C_{3} \times C_{3} \times C_{3}$ and $\gamma_{3}(G)=1$ follows easily as $t\left(G^{\prime}\right)=7$ and $\delta^{(2)}(K G)=\Delta\left(G^{\prime}\right)^{3} K G$.

Example 2.4. By above Theorem $\delta^{(3)}\left(K D_{10}\right)=0$, if Char $K=5$ where $D_{10}$ denotes the Dihedral group of order 10. Thus in this case $G^{\prime}=C_{5}$ need not be central and $G$ need not even be nilpotent. Also if $G$ is the semidirect product of $C_{3} \times C_{3}$ by $C_{2}$ induced by the automorphism sending every element of $C_{3} \times C_{3}$ to its inverse, then $\delta^{(3)}(K G)=0$ where Char $K=3$. Thus in Char $K=3$ also $G$ need not be nilpotent.

Corollary 2.5. Let $K$ be a field with Char $K=p \geq 7$ and let $G$ be a group. Then the following are equivalent:

(i) $\delta^{(3)}(K G)=0$,

(ii) $\delta^{[3]}(L(K G))=0$.

Proof: Clearly if $\delta^{(3)}(K G)=0$, then $\delta^{[3]}(L(K G))=0$. Suppose that $\delta^{[3]}(L(K G))=0$. Let $x, y \in G$. Then by $[\mathbf{6}$, Lemma 2.4(iii)] $2((x, y, y)-1)^{3} \in \gamma_{3}\left(\delta^{[1]}(L(K G))\right)$. Since by [5, Lemma 1.7] $\left[\gamma_{3}\left(\delta^{[1]}(L(K G))\right)\right]^{2} K G \subseteq \delta^{[3]}(L(K G)) K G$, we get that $4((x, y, y)-1)^{6}=0$. Since $G^{\prime}$ is a $p$-group and $p \geq 7,(x, y, y)=1$ and $G$ is 2-Engel. It is well known that for a 2-Engel group $G,\left(G^{\prime}, G\right)^{3}=1$. Again because $G^{\prime}$ is a $p$-group, $p \geq 7$, we conclude that $\gamma_{3}(G)=1$. Now $[[x, y][x, y, y],[x, y]]=[[x, y],[x y, y],[x, y]]$ is in $\gamma_{3}\left(\delta^{[1]}(L(K G))\right)$. Therefore as above

$$
\begin{aligned}
0 & =[[x, y][x, y, y],[x, y]]^{2} \\
& =[y x((x, y)-1)[y x((x, y)-1), y], y x((x, y)-1)]^{2} \\
& =[y x y[x, y], y x]^{2}((x, y)-1)^{6} \\
& =\left[y x y^{2} x((x, y)-1), y x\right]^{2}((x, y)-1)^{6} \\
& =\left[y x y^{2} x, y x\right]^{2}((x, y)-1)^{8} \\
& =\left(y x y^{2} x y x\right)^{2}((x, y)-1)^{10} .
\end{aligned}
$$

This gives that $((x, y)-1)^{10}=0$. But $G^{\prime}$ is a $p$-group and hence for $p \geq 11,(x, y)=1$ and for $p=7,(x, y)^{7}=1$. We conclude that for $p \geq 11, G$ is abelian and for $p=7, G^{\prime 7}=1$. Now let $p=7$ and let $x, y$, $u, v \in G$, then

$$
\begin{aligned}
{[[x, y][x, y, y],[x, y]][[u, v][u, v, v],[u, v]] } & \in\left[\gamma_{3}\left(\delta^{[1]}(L(K G))\right)\right]^{2} \\
& \subseteq \delta^{[3]}(L(K G))=0 .
\end{aligned}
$$


Simplifying as above we get $((x, y)-1)^{5}((u, v)-1)^{5}=0$. And so $(u, v) \in$ $\langle(x, y)\rangle$. Thus $G^{\prime}$ is cyclic. Rest follows from Theorem 2.3.

Next we give an example to illustrate that there are group algebras which are Lie solvable of length three but not strongly Lie solvable of length three.

Example 2.6. Let $K=\mathbb{Z}_{2}$ and $G=S_{3}$, the symmetric group on three letters. Then $G^{\prime}$ is a cyclic group of order three. It can be easily verified that $\delta^{[2]}(L(K G)) \subseteq K G^{\prime}$ and hence $\delta^{[3]}(L(K G))=0$. But $\mathbb{Z}_{2} S_{3}$ is not strongly Lie solvable.

Acknowledgements. The author is thankful to the referee for valuable suggestions.

\title{
References
}

1. F. Levin and G. Rosenberger, Lie metabelian group rings, Preprint no. 60, Ruhr-Universität, Bochum, Dec. 1985.

2. K. Motose and Y. Ninomiya, On the nilpotency index of the radical of a group algebra, Hokkaido Math. J. 4 (1975), 261-264.

3. M. Sahai and J. B. Srivastava, A note on Lie centrally metabelian group algebras, to appear in J. Algebra.

4. S. K. Sehgal, "Topics in Group Rings," Marcel Dekker, 1978.

5. R. K. Sharma and J. B. Srivastava, Lie solvable rings, Proc. Amer. Math. Soc. 94 (1985), 1-8.

6. R. K. Sharma and J. B. SRivastava, Lie centrally metabelian group rings, J. Algebra 151(2) (1992), 476-486.

\author{
Department of Mathematics \\ Indian Institute of Technology \\ New Delhi - 110016 \\ INDIA
}

Primera versió rebuda el 6 de Juliol de 1994, darrera versió rebuda el 29 de Juny de 1995 